\title{
Effects of Magnetic Field on the MHD Flow of a Third Grade Fluid through Inclined Channel with Ohmic Heating
}

\section{Aiyesimi YM1 ${ }^{1}$, Okedayo $\mathrm{GT}^{2}$ and Lawal OW ${ }^{3 *}$}

${ }^{1}$ Department of Mathematics, Federal University of Technology Minna, Niger State, Nigeria

${ }^{2}$ Department of Mathematics, Ondo State University of Science and Technology Okitipupa, Ondo State, Nigeria

${ }^{3}$ Department of Mathematics, Tai Solarin University of Education ljagun, Ogun State, Nigeria

\begin{abstract}
In this paper, we investigate the combine effects of magnetic field on the MHD flow of a third grade fluid through inclined channel in the presence of a uniform magnetic field with the consideration of heat transfer. Three different problems, Couette flow, Poiseuille flow and Couette-Poiseuille flow have been analysed. The non-linear differential equations governing the flow and heat transfer are solved for the velocity and temperature profile by employing the regular perturbation technique and the results are presented graphically.
\end{abstract}

Keywords: Couette flow; Poiseuille flow; Couette-Poiseuille flow; Third grade fluid; Regular perturbation method; Magnetohydrodynamics; Inclined channel

\section{Introduction}

In recent years considerable interest has been developed in the study of the flow of third grade fluid through an inclined parallel plate because of its important applications in science, engineering and technology. Some of these applications can be found in materials manufactured by extraction process especially in polymer processing, micro fluids, geological flows within the earth's mantle, the flow of synovial fluid in human joints as well as in the drilling of oil and gas wells. Nayak et al. [1] study the unsteady convective flow of a third grade fluid past an infinite vertical porous plate with uniform suction applied at the plate. They reduced governing non linear partial differential equations arising from the problem to a system of nonlinear algebraic equations using implicit finite difference scheme and is then solved these equations using derived Newton method. Erdogan [2] considered the flow of a third grade fluid in the vicinity of a plane wall suddenly set in motion. He observes that for short time a strong non-Newtonian effect is present in the velocity field. For more list of references concerning this work, we refer to the articles by Hayat et al. [3] and Roohi et al. [4], But Siddidui et al. [5] studied hydrodynamics third grade fluid between two parallel plates with heat transfer. They considered and treated three different problems, the Poiseuille flow, the Couette flow and the Poiseuille-Couette flow. The governing nonlinear differential equations for velocity and temperature of the fluid were also derived and solved using homotopy perturbation method. In this work, we extend the work of Siddiqui et al. [5] in two ways (i) to consider that the fluid is flowing down through inclined parallel plates and (ii) subjected the whole system into the magnetic field and analyze the effect of magnetic field on MHD flow.

\section{Problem Formulation}

The fundamental equations governing the MHD flow of an incompressible electrically conducting fluid are the field equation:

$$
\begin{aligned}
& \nabla \cdot v=0 \\
& \rho \frac{D v}{D t}=-\nabla p+\operatorname{div} \tau+J \times B+\rho f
\end{aligned}
$$

where $\rho$ is the density $\mathrm{f}$ the fluid, $v$ is the fluid velocity, $B$ is the magnetic induction so that

$$
B=B_{0}+b
$$

and

$$
J=\sigma(E+v \times B)
$$

is the current density, $\sigma$ is the electrical conductivity, $E$ is the electrical filed which is not considered (i.e $E=0$ ), $B_{0}$ and $b$ are applied and induced magnetic field respectively, $D / D$ tenote the material derivative, $\mathrm{p}$ is the pressure, $\mathrm{f}$ is the external body force and $\mathrm{T}$ is the Cauchy stress tensor which for a third grade fluid satisfies the constitutive equation

$$
\begin{gathered}
\tau=-p I+\mu A_{1}+\alpha_{1} A_{2}+\alpha_{2} A_{1}^{2}+\beta_{1} A_{3} \\
+\beta_{2}\left(A_{1} A_{2}+A_{2} A_{1}\right)+\beta_{3}\left(t r A_{1}^{2}\right) A_{1} \\
A_{n}=\frac{D A_{n-1}}{D t}+A_{n-1} \nabla v+(\nabla v)^{\perp} A_{n-1}, n \geq 1
\end{gathered}
$$

where $p I$ is the isotropic stress due to constraint incompressibility, $\mu$ is the dynamics viscosity, $\alpha_{1}, \alpha_{2}, \beta_{1}, \beta_{2}, \beta_{3}$ are the material constants; $\perp$ indicate the matrix transpose, $A_{1}, A_{2}, A_{3}$ are the first three RivlinEricken tensors and $A_{0}=I$ is the identity tensor [6-11].

\section{Couette flow}

We consider a steady MHD flow of a third grade fluid through a infinite parallel plates of distant $2 h$ apart, inclined horizontally by angle $\phi$. The upper and lower plates are at $y=\mathrm{h}$ and $\mathrm{y}=-\mathrm{h}$ of a rectangular system with the $x$-axis as flow direction. The upper plate is assumed to be moving with constant speed $U$ while the lower plate is kept stationary. The plates are maintained at two different but constant temperatures with upper plate simultaneously subjected to a step change in temperature $\Delta T$. A uniform magnetic field $B_{0}$ is applied in positive $y$-direction and is assumed undisturbed as the induced

*Corresponding author: Lawal OW, Department of Mathematics, Tai Solarin University of Education ljagun, Ogun State, Nigeria, Tel: 07032270305, 234(0)7098809476; E-mail: waheedlawal207@yahoo.com

Received January 17, 2014; Accepted February 14, 2014; Published February 14,2014

Citation: Aiyesimi YM, Okedayo GT, Lawal OW (2014) Effects of Magnetic Field on the MHD Flow of a Third Grade Fluid through Inclined Channel with Ohmic Heating. J Appl Computat Math 3: 153. doi:10.4172/2168-9679.1000153

Copyright: (c) 2014 Aiyesimi YM, et al. This is an open-access article distributed under the terms of the Creative Commons Attribution License, which permits unrestricted use, distribution, and reproduction in any medium, provided the original author and source are credited. 
magnetic field is neglected under the assumption of small magnetic Reynolds number. The ambient air is assumed stationary so that the flow is due to movement of upper plate and gravity alone.

By seeking a velocity field of the form

$$
v=[u(y), 0,0,]
$$

In the absence of modified pressure gradient, equation (1)-(4) along with equation (5)-(7) after introducing the following non dimensional parameter

$$
u=U \bar{u}, y=\bar{y} h, \bar{T}=\frac{T-T_{0}}{\Delta T}
$$

yield

$$
\frac{\partial^{2} u}{\partial y^{2}}+6 \beta\left(\frac{\partial u}{\partial y}\right)^{2} \frac{\partial^{2} u}{\partial y^{2}}+K-M u=0
$$

with the boundary conditions

$$
u(-1)=0, u(1)=1
$$

Also, thethermalboundarylayerequation for thethermodynamically compatible third grade fluid with viscous dissipation, work done due to deformation and joule heating in a non dimensional form is given as

$$
\frac{d^{2} T}{d y^{2}}+B_{r}\left(\frac{d u}{d y}\right)^{2}+2 B_{r} \beta\left(\frac{d u}{d y}\right)^{4}+B_{r} M u^{2}=0
$$

with the boundary

$$
T(-1)=0, T(1)=1
$$

where

$\beta=\frac{\left(\beta_{2}+\beta_{3}\right)}{\mu}\left(\frac{U}{h}\right)^{2}$ is third grade fluid parameter

$K=f_{1} \sin \phi$ while $f_{1}=\frac{h^{2} \rho g}{\mu U}$ is the gravitational parameter.

$M=\frac{h^{2} \sigma B_{0}^{2}}{\mu}$ is the magnetic parameter.

$B_{r}=\frac{\mu U^{2}}{k \Delta T}$ is the Brinkman number

\section{Poiseuille flow}

In this case, both the upper and lower plates are kept stationary and we assumed that the fluid motion is due to gravity alone.

Therefore, in the absence of modified pressure gradient, equation (9) and (11) remain the same after scaling given by (8) while boundary conditions (10) and (12) becomes

$$
\begin{aligned}
& u(-1)=0, u(1)=0 \\
& T(-1)=0, T(1)=1
\end{aligned}
$$

\section{Couette-poiseuille flow}

Because we considered that the flow is due to gravity and the movement of upper plate while the modified pressure gradient is neglected, the momentum and energy equations with their boundary conditions for Couette-Poiseuille flow will result to that of the Couette flow.

Therefore, we solve problem in subsection (2.1) and (2.2) by traditional perturbation method while the problem involved in subsection (2.3) is identical to the problem in subsection (2.1) because of our assumption.

\section{Solution of the Problems}

\section{Couette flow}

Let us assume $\varepsilon$ as a small parameter in order to solve equation (9) with equation (10), we expand

$$
u(y, \varepsilon)=u_{0}(y)+\varepsilon u_{1}(y)+\varepsilon^{2} u_{2}(y)+\cdots \cdots
$$

We first find the solution for section (2.1) (i.e Couette flow) by substituting equation (15) into equation (9) and (10) and rearranging based on powers of $\varepsilon$-terms.

We obtain the following problems of different order with their boundary conditions and solutions:

Zeroth-order problem

$$
\begin{aligned}
& \frac{d^{2} u_{0}}{d y^{2}}-M u+K=0 \\
& u_{0}(-1)=0, u_{0}(1)=1
\end{aligned}
$$

with the solution

where

$$
u_{0}=c_{1} e^{y \sqrt{M}}+c_{2} e^{-y \sqrt{M}}+\frac{K}{M}
$$

$$
c_{1}=-\frac{\left(e^{\sqrt{M}} M+e^{-\sqrt{M}} K-K e^{\sqrt{M}}\right)}{M\left(-\left(e^{\sqrt{M}}\right)^{2}+\left(e^{-\sqrt{M}}\right)^{2}\right)} c_{2}=-\frac{\left(-e^{-\sqrt{M}} M+e^{-\sqrt{M}} K-K e^{\sqrt{M}}\right)}{M\left(-\left(e^{\sqrt{M}}\right)^{2}+\left(e^{-\sqrt{M}}\right)^{2}\right)}
$$

First order problem

$$
\begin{aligned}
& \frac{d^{2} u_{1}}{d y^{2}}+6 \frac{d^{2} u_{0}}{d y^{2}}\left(\frac{d u_{0}}{d y}\right)^{2}-M u_{1}=0 \\
& u_{1}(-1)=0, u_{1}(1)=0
\end{aligned}
$$

with solution

$$
\begin{aligned}
u_{1}= & c_{3} e^{3 y \sqrt{M}}+c_{4} e^{-3 y \sqrt{M}}+\left(c_{5}+c_{6} y\right) e^{y \sqrt{M}} \\
& +\left(c_{7}+c_{8} y\right) e^{-y \sqrt{M}}
\end{aligned}
$$

Second order problem

$$
\begin{aligned}
& \frac{d^{2} u_{2}}{d y^{2}}+6 \frac{d^{2} u_{1}}{d y^{2}}\left(\frac{d u_{0}}{d y}\right)^{2}+12 \frac{d u_{0}}{d y} \frac{d u_{1}}{d y} \frac{d^{2} u_{0}}{d y^{2}} \\
& -M u_{2}=0 \\
& u_{2}(-1)=0, u_{2}(1)=0
\end{aligned}
$$

with solution

$$
\begin{aligned}
u_{2}= & c_{19} e^{5 y \sqrt{M}}+c_{20} e^{-5 y \sqrt{M}}+ \\
& \left(c_{21}+c_{22} y\right) e^{3 y \sqrt{M}}+\left(c_{23}+c_{24} y\right) e^{-3 y \sqrt{M}} \\
& +\left(c_{25}+c_{26} y+c_{27} y^{2}\right) e^{y \sqrt{M}} \\
& +\left(c_{28}+c_{29} y+c_{30} y^{2}\right) e^{-y \sqrt{M}}
\end{aligned}
$$


By using equation (18), (21) and (24) in equation (15), we obtain

$$
\begin{aligned}
u(y)= & c_{1} e^{y \sqrt{M}}+c_{2} e^{-y \sqrt{M}}+\frac{K}{M} \\
& +\varepsilon\left(\begin{array}{l}
c_{3} e^{3 y \sqrt{M}}+c_{4} e^{-3 y \sqrt{M}} \\
+\left(c_{5}+c_{6} y\right) e^{y \sqrt{M}} \\
+\left(c_{7}+c_{8} y\right) e^{-y \sqrt{M}}
\end{array}\right) \\
& +\varepsilon^{2}\left(\begin{array}{l}
c_{19} e^{5 y \sqrt{M}}+c_{20} e^{-5 y \sqrt{M}} \\
+\left(c_{21}+c_{22} y\right) e^{3 y \sqrt{M}} \\
+\left(c_{23}+c_{24} y\right) e^{-3 y \sqrt{M}} \\
+\left(c_{25}+c_{26} y+c_{27} y^{2}\right) e^{y \sqrt{M}} \\
+\left(c_{28}+c_{29} y+c_{30} y^{2}\right) e^{-y \sqrt{M}}
\end{array}\right)
\end{aligned}
$$

Next, we find the approximate solution for temperature distribution, for which we write

$$
T(y, \varepsilon)=T_{0}(y)+\varepsilon T_{1}(y)+\varepsilon^{2} T_{2}(y)+\cdots
$$

Substituting equation (26) into equation (11)-(12) and collecting the same power of $\varepsilon$, yields different order problems with boundary conditions and solutions

Zeroth -order problem

$$
\begin{aligned}
& \frac{d^{2} T_{0}}{d y^{2}}+B_{r}\left[\left(\frac{d u_{0}}{d y}\right)^{2}+M u_{0}\right]=0 \\
& T_{0}(-1)=0, T_{0}(1)=1
\end{aligned}
$$

with solution

$$
\begin{aligned}
T_{0}= & c_{31} e^{2 y \sqrt{M}}+c_{32} e^{-2 y \sqrt{M}}+c_{33} e^{y \sqrt{M}} \\
& +c_{34} e^{-y \sqrt{M}}+c_{35} y^{2}+c_{36} y+c_{37}
\end{aligned}
$$

First-order problem

$$
\frac{d^{2} T_{1}}{d y^{2}}+2 B_{r}\left[\frac{d u_{0}}{d y} \frac{d u_{1}}{d y}+\left(\frac{d u_{0}}{d y}\right)^{4}+M u_{0} u_{1}\right]=0
$$

$T_{1}(-1)=0, T_{1}(1)=0$

with solution

$$
\begin{aligned}
T_{1}= & c_{51} e^{4 y \sqrt{M}}+c_{52} e^{-4 y \sqrt{M}}+c_{53} e^{3 y \sqrt{M}} \\
& +c_{54} e^{-3 y \sqrt{M}}+\left(c_{55}+c_{56} y\right) e^{2 y \sqrt{M}} \\
& +\left(c_{57}+c_{58} y\right) e^{-2 y \sqrt{M}}+\left(c_{59}+c_{60} y\right) e^{y \sqrt{M}} \\
& +\left(c_{61}+c_{62} y\right) e^{-y \sqrt{M}}+c_{63} y^{2}+c_{64} y+c_{65}
\end{aligned}
$$

Second-order problem

$$
\frac{d^{2} T_{2}}{d y^{2}}+B_{r}\left[\begin{array}{l}
2 \frac{d u_{0}}{d y} \frac{d u_{2}}{d y}+\left(\frac{d u_{1}}{d y}\right)^{2} \\
+8\left(\frac{d u_{0}}{d y}\right)^{3} \frac{d u_{1}}{d y} \\
+2 M u_{0} u_{2}+M u_{1}^{2}
\end{array}\right]=0
$$

$$
T_{2}(-1)=0, T_{2}(1)=0
$$

with the solution

$$
\begin{aligned}
T_{2}= & c_{93} e^{6 y \sqrt{M}}+c_{94} e^{-6 y \sqrt{M}}+c_{95} e^{5 y \sqrt{M}} \\
& +c_{96} e^{-5 y \sqrt{M}}+\left(c_{97}+c_{98} y\right) e^{4 y \sqrt{M}} \\
& +\left(c_{99}+c_{100} y\right) e^{-4 y \sqrt{M}}+\left(c_{101}+c_{102} y\right) e^{3 y \sqrt{M}} \\
& +\left(c_{103}+c_{104} y\right) e^{-3 y \sqrt{M}} \\
& +\left(c_{105}+c_{106} y+c_{107} y^{2}\right) e^{2 y \sqrt{M}} \\
& +\left(c_{108}+c_{109} y+c_{110} y^{2}\right) e^{-2 y \sqrt{M}} \\
& +\left(c_{111}+c_{112} y+c_{113} y^{2}\right) e^{y \sqrt{M}} \\
& +\left(c_{114}+c_{115} y+c_{116} y^{2}\right) e^{-y \sqrt{M}}+c_{117} y^{4} \\
& +c_{118} y^{3}+c_{119} y^{2}++c_{120} y+c_{121}
\end{aligned}
$$

By using equation (29), (32) and (35) in equation (26), we obtain

$$
\begin{aligned}
T(y)= & c_{31} e^{2 y \sqrt{M}}+c_{32} e^{-2 y \sqrt{M}}+c_{33} e^{y \sqrt{M}} \\
+ & c_{34} e^{-y \sqrt{M}}+c_{35} y^{2}+c_{36} y+c_{37} \\
& \left(\begin{array}{l}
c_{51} e^{4 y \sqrt{M}}+c_{52} e^{-4 y \sqrt{M}}+ \\
c_{53} e^{3 y \sqrt{M}}+c_{54} e^{-3 y \sqrt{M}} \\
+\left(c_{55}+c_{56} y\right) e^{2 y \sqrt{M}} \\
+\left(c_{57}+c_{58} y\right) e^{-2 y \sqrt{M}} \\
+\left(c_{59}+c_{60} y\right) e^{y \sqrt{M}} \\
+\left(c_{61}+c_{62} y\right) e^{-y \sqrt{M}} \\
+c_{63} y^{2}+c_{64} y+c_{65}
\end{array}\right)
\end{aligned}
$$

$$
+\varepsilon^{2}\left(\begin{array}{l}
c_{93} e^{6 y \sqrt{M}}+c_{94} e^{-6 y \sqrt{M}} \\
+c_{95} e^{5 y \sqrt{M}}+c_{96} e^{-5 y \sqrt{M}} \\
+\left(c_{97}+c_{98} y\right) e^{4 y \sqrt{M}} \\
+\left(c_{99}+c_{100} y\right) e^{-4 y \sqrt{M}} \\
+\left(c_{101}+c_{102} y\right) e^{3 y \sqrt{M}} \\
+\left(c_{103}+c_{104} y\right) e^{-3 y \sqrt{M}} \\
+\left(c_{105}+c_{106} y+c_{107} y^{2}\right) e^{2 y \sqrt{M}} \\
+\left(c_{108}+c_{109} y+c_{110} y^{2}\right) e^{-2 y \sqrt{M}} \\
+\left(c_{111}+c_{112} y+c_{113} y^{2}\right) e^{y \sqrt{M}} \\
+\left(c_{114}+c_{115} y+c_{116} y^{2}\right) e^{-y \sqrt{M}} \\
+c_{117} y^{4} \\
+c_{118} y^{3}+c_{119} y^{2}++c_{120} y+c_{121}
\end{array}\right)
$$

where the constant $c_{3}-c_{121}$ can be calculated through simple computation.

\section{Poiseuille flow}

Next we consider approximate solutions for problem in section (2.2). We used momentum equation (9) and energy equation (11) 
with boundary conditions (13) and (14) respectively. By substituting equation (15) and (16) into equation (9) and (11) respectively, we obtained different order problems which are identical to those in section (3.1) except the boundary conditions (17) of Zeroth order problem of equation (9) which becomes

$$
u_{0}(-1)=0, u_{0}(1)=0
$$

The solutions obtained for problem in section (2.2) (i.e. Poiseuille flow) are identical to those in section (3.1) (i. e. couette flow) except

$$
c_{1}=c_{2}=-\frac{K}{\left(e^{\sqrt{M}}+e^{-\sqrt{M}}\right) M}
$$

\section{Couette-Poiseuille flow}

Since the problem involved in subsection (2.3) (i.e CouettePoiseuille flow) is identical to the problem in subsection (2.1) (i.e Couette flow) base on our assumption, we obtained the same solution.

\section{Results and Discussion}

We shall proceed to discuss three flow problems namely, Couette

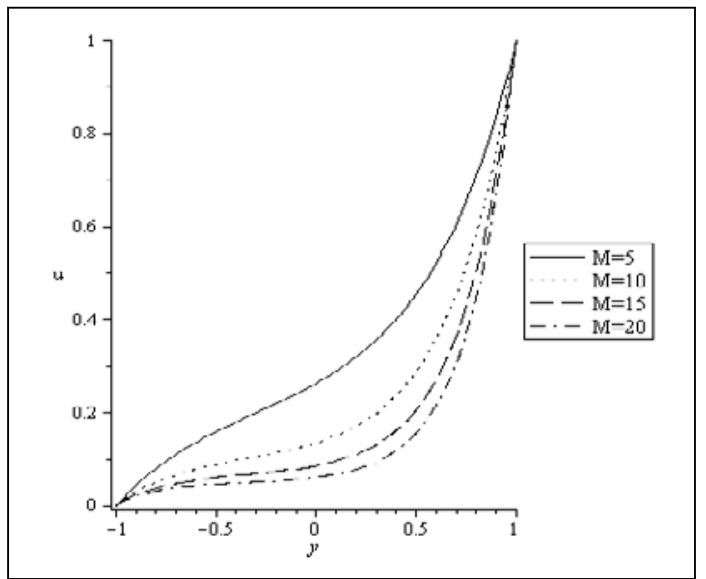

(a)

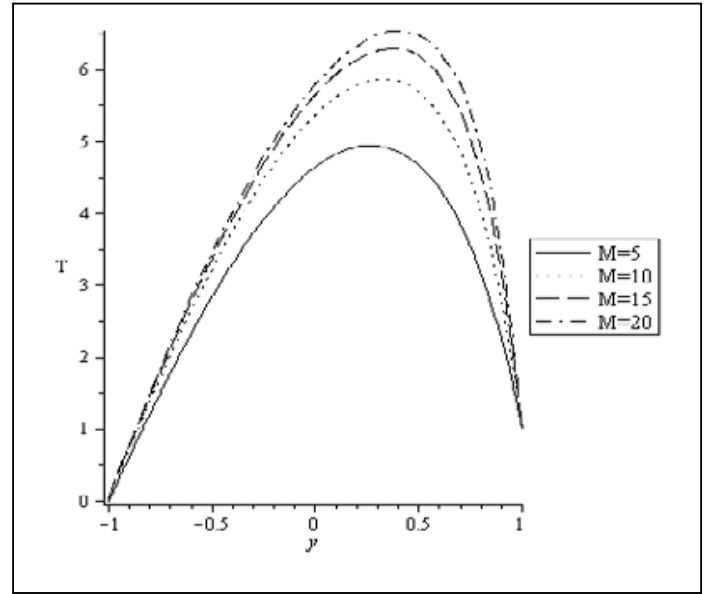

(b)

Figure 1: Effect of magnetic parameter $M$ on velocity and temperature profile of Couette flow when $K=1, B r=5$ and $\varepsilon=0.001$. (a)

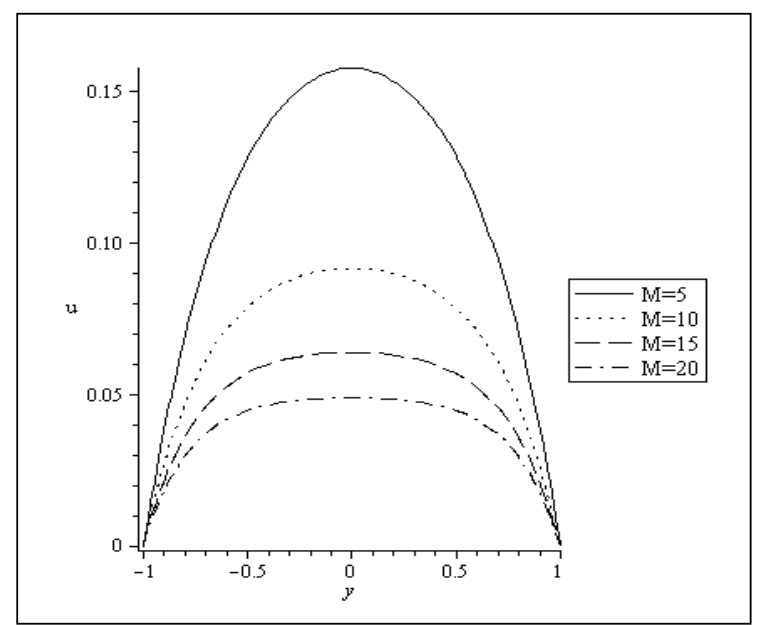

(b)

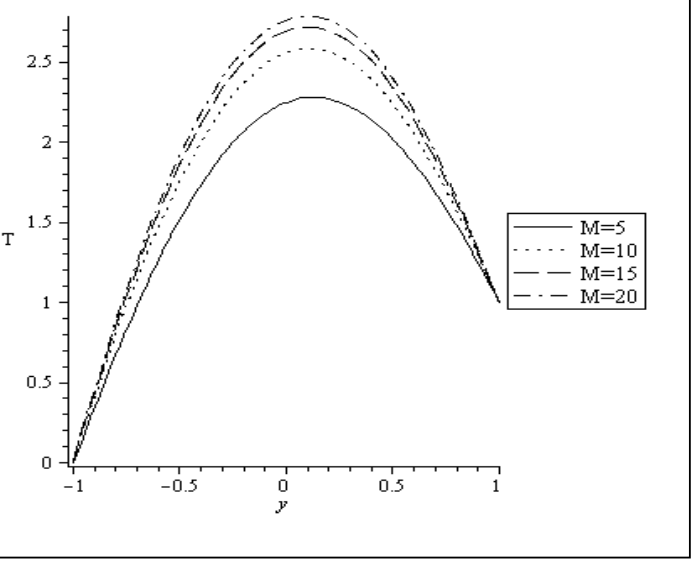

Figure 2: Effect of magnetic parameter $M$ on Velocity and temperature profile of Poiseuille flow when $K=1, B r=5$ and $\varepsilon=0.001$.

flow, Poiseuille flow and Couette-Poiseuille flow of a third grade fluid between two inclined parallel plates with heat transfer. Also, both Couette flow and Couette-Poiseuille flow have the same solution base on our assumption. Therefore we discussed and shown graphically (Figures 1-6) the effects of magnetic field and gravitational parameter on velocity and temperature profile of Couette and Poiseuille flow.

In Figure 1a (i.e Couette flow), it is observed that fluid velocity decreases with increase in magnetic parameter $M$ and the rate at which the flow of the fluid decreases is less apparent near the lower plate to the upper plate. This is due to the fact that the rate of transport of the fluid is considerably reduced near the moving upper plate than the stationary lower plate as the magnetic parameter increases. In Poiseuille flow (i.e. Figure $2 \mathrm{a}$ ), velocity of the fluid decreases with increases in magnetic field parameter and the flow of the fluid decreases equally from the stationary lower plate to the stationary upper plate. It clearly shows that the transverse magnetic field opposes the transverse phenomena. Figures $1 \mathrm{~b}$ and $2 \mathrm{~b}$ illustrates the graphical representation of the temperature profiles $T$ for various values of physical parameters. It is noticed from figures that the fluid temperature increases with increases in magnetic parameter $M$. And the rate at which the temperature of the fluid increases is less apparent from the stationary lower plate to the 
(a)

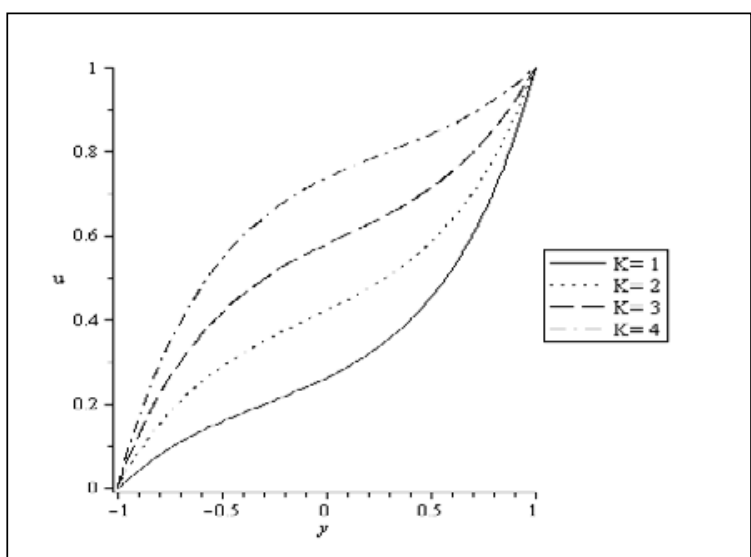

(b)

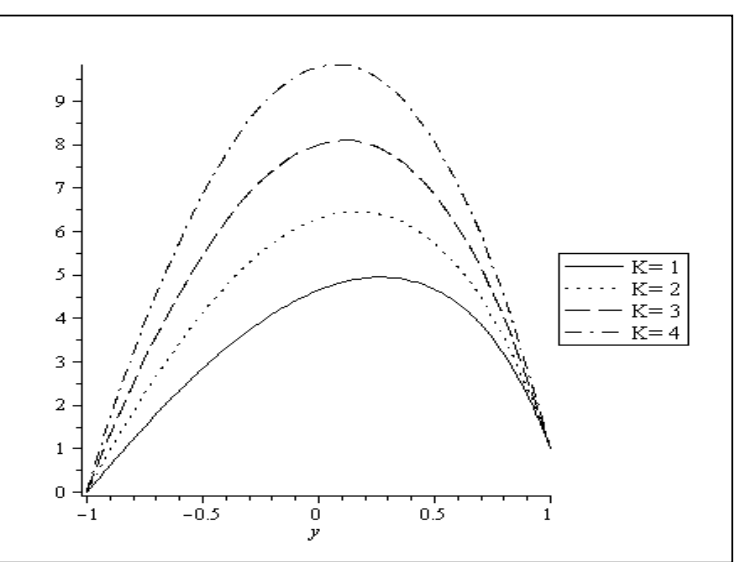

Figure 3: Effect of gravitational parameter $K$ on velocity and temperature profile of Couette flow when $M=5, B r=5$ and $\varepsilon=0.001$

moving upper plate in Figure 1b. This clearly shows that an increase in magnetic parameter lead to increase on Joule heating thereby increases the temperature of the fluid.

Figures 3 and 4 shows the effect of gravitational parameter $K$ on velocity and temperature profile at a low magnetic field (i.e. when $M=5)$. It is observed that the velocity increases rapidly from the moving upper plate to the stationary lower plate in Figure 3a while in Figure $4 \mathrm{a}$, the velocity of the fluid increases through the two stationary plates as gravitational parameter $K$ increases. However, in Figures $3 \mathrm{~b}$ and $4 \mathrm{~b}$ the temperature rapidly rises with increase in gravitational parameter $K$ and is higher in Figure $3 b$ due to the fact that in Couette flow the movement of upper plate increases the fluid velocity thereby increases Joule dissipation.

Figures 5 and 6 illustrate the effect of gravitational parameter $K$ on velocity and temperature profile at a high magnetic field (i.e when $M=20$ ). It is observed that the rate at which the velocity increases reduced rapidly from the moving upper plate to the stationary lower plate in Figure $5 \mathrm{a}$ while in Figure 6a, the rate at which the velocity of the fluid increases through the two stationary plates is reduced as gravitational parameter $K$ increases. However, in Figures $5 b$ and $6 \mathrm{~b}$ the temperature rapidly increases with increase in gravitational parameter K. (a)

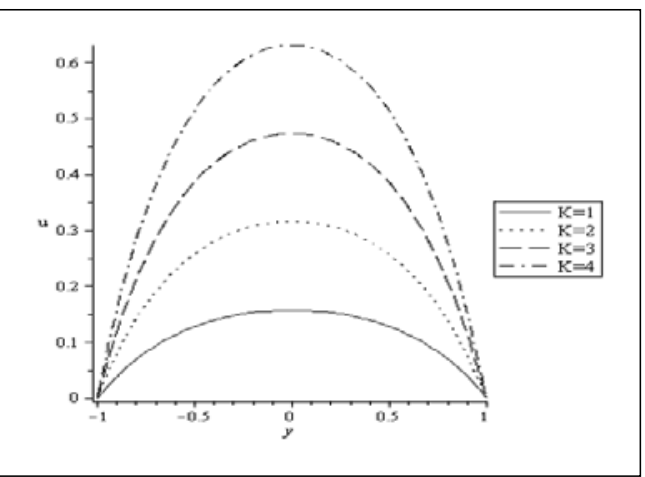

(b)

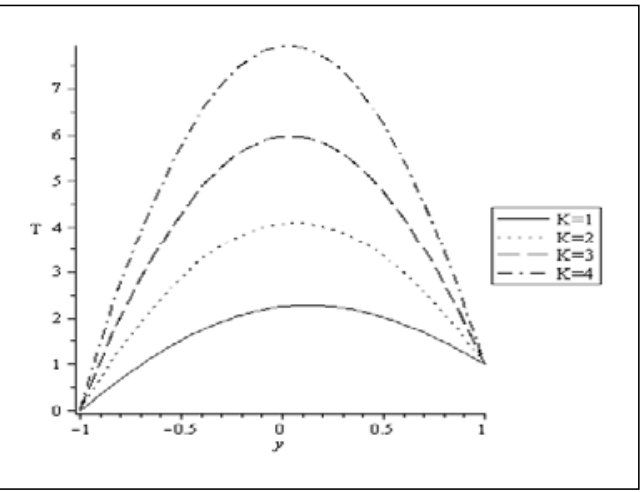

Figure 4: Effect of gravitational parameter $K$ on velocity and temperature profile of Poiseuille flow when $M=5, B r=5$ and $\varepsilon=0.001$.

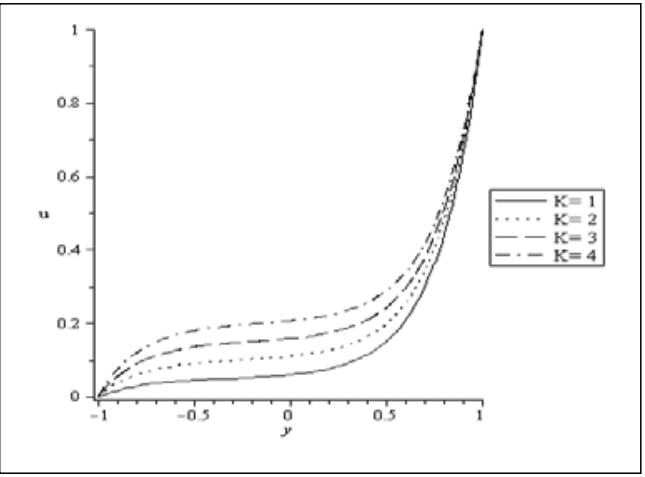

(a)

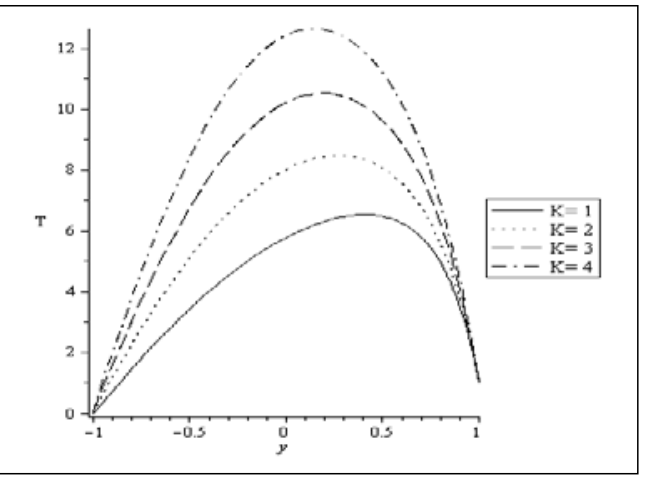

Figure 5: Effect of gravitational parameter $K$ on velocity and temperature profile of Couette flow when $M=20, B r=5$ and $\varepsilon=0.001$. 
Citation: Aiyesimi YM, Okedayo GT, Lawal OW (2014) Effects of Magnetic Field on the MHD Flow of a Third Grade Fluid through Inclined Channel with Ohmic Heating. J Appl Computat Math 3: 153. doi:10.4172/2168-9679.1000153

Page 6 of 6

(a)

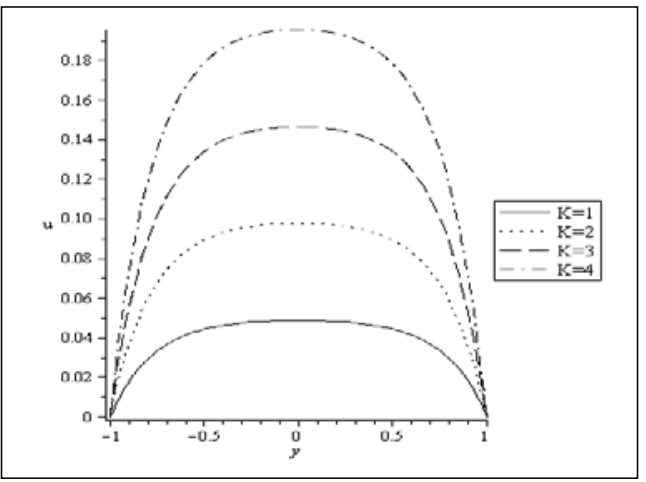

(b)

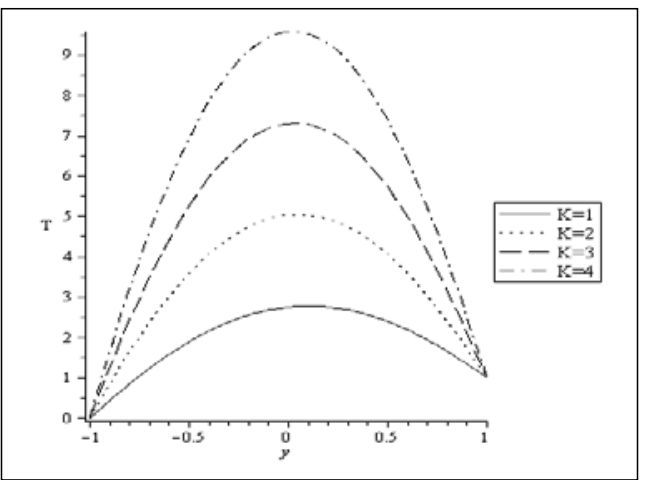

Figure 6: Effect of gravitational parameter $K$ on velocity and temperature profile of Poiseuille flow when $M=20, B r=5$ and $\varepsilon=0.001$.

\section{Conclusion}

In this paper, the solution for the velocity and temperature profile of a third grade MHD fluid flow between two inclined parallel plates with heat transfer was approximated analytically. The governing nonlinear differential equations involved are solved using the traditional perturbation method. The effects of magnetic and gravitational parameters on velocity and temperature profile for Couette and Poiseuille flows have been discussed and are shown graphically.
The following conclusion can be drawn from the computed results:

- The effect of magnetic field parameter is to decrease velocity profile and increase the temperature profile in the boundary layer as fluid is flowing down through the plates.

- The effect of gravitation parameter is to increase the velocity and temperature profile at low magnetic field and decrease them at high magnetic field.

-We can regain the results of Newtonian fluid when $\beta=0$.

\section{References}

1. Nayak I, Nayak AK, Padhy S (2012) "Numerical Solution for the flow and heat transfer of a third grade fluid past a porous vertical plate, "Adv Studies Theor Phys 6: 615-624.

2. Erdogan ME (1995) Plane surface suddenly set into motion in a non-Newtonian fluid, Acta mech 108: 179-187.

3. Hayat T, Ellahi R, Mahomed F (2009) "The analytical solutions for Magnetohydrodynamic flow of a third order fluid in a porous medium", Z Naturforsch 64: 531-539.

4. Roohi E, Khara S, Farjami Y (2009) "Application of the homotopy method for analytical solution of non-Newtonian channel flows", Phys Scr 79.

5. Siddiqui AM, Zeb A, Ghori QK, Benhartbit AM (2008) "Homotopy Perturbation Method for heat transfer flow of a third grade fluid between parallel plate". Chaos solitons and fractals 36: 182-192.

6. Rivlin RS, Ericksen JL (1995) Stress deformation relations for isotropic materials, J Rat Mech impass Anal 3: 323.

7. Ellehi R (2009) Effects of the slip boundary condition on non-Newtonian flows in a channel. Commu Nonlinear Science Numerical Simul 14: 1377-1384.

8. Aiyesimi YM, Okedayo, GT, Lawal OW (2013) Viscous dissipation effect on the MHD flow of a third grade fluid down an inclined plane with Ohmic heating. Mathematical Theory and Modelling 9: 133-166.

9. Hayat T, Saif $S$, Abbas $Z$ (2008) The influence of heart transfer in an MHD second grade fluid film over an unsteady stretching sheet. Physics Letters $A$ 372: 5037-5045.

10. Makinde OD (2009) "Thermal critically for a reactive gravity driven thin film flow of a third grade fluid with adiabatic free surface down an inclined plane". Appl Math Mech Ed 30: 373-380.

11. Sajid M, Awals M, Nadeem S, Hagat T (2008) "The influence of slip condition on thin film flow of a fourth grade fluid by the homotopy analysis method". Comput Math Appl 56: 2019-2026. 